ARTICULOS ORIGINALES

Rev Chil Salud Pública 2020,

Vol 24(2) 97-103

\section{USO DE CONDÓN EN ESTUDIANTES DE PREGRADO DE CARRE- RAS SANITARIAS DE LA UNIVERSIDAD DE VALPARAÍSO: ESTUDIO TRANSVERSAL}

\author{
USE OF CONDOMS AMONG UNDERGRADUATE HEALTH STUDENTS \\ AT UNIVERSIDAD DE VALPARAÍSO: A CROSS-SECTIONAL STUDY
}

\section{RESUMEN}

Introducción: El uso de condones masculinos permite la prevención de infecciones de transmisión sexual y de embarazo, pero su uso en estudiantes de carreras sanitarias ha sido poco estudiado en Chile. El objetivo del presente estudio es evaluar el uso de condón y caracterizar la actividad sexual y el antecedente de infección de transmisión sexual en estudiantes de la Facultad de Medicina de la Universidad de Valparaíso, Chile. Materiales y métodos: Se desarrolló un estudio transversal. Se aplicó el Cuestionario Confidencial Sobre Vida Sexual Activa. Para el análisis inferencial se usaron las pruebas de Fisher y Kruskal-Wallis. Resultados: Se incluyeron 143 estudiantes (69\% mujeres). El promedio de edad de la primera relación sexual fue de 17+1,8 años. Durante los últimos 12 meses, 6,3\% siempre uso condón en las relaciones sexuales penetrativas, $69,2 \%$ a veces y $24,5 \%$ nunca $(91,6 \%$ de ellas fueron heterosexuales); de acuerdo con el tipo de práctica sexual, 41,9\% declaró siempre usar condón en las relaciones anales, 25,8\% en las vaginales y 0,8\% en las orales; 3,5\% había presentado alguna infección de transmisión sexual. No se encontraron asociaciones entre el uso de condón y el sexo de los participantes, el antecedente de infección de transmisión sexual, el promedio de edad de inicio de la actividad sexual o la carrera universitaria. Discusión: Un pequeño número de participantes reportó el antecedente de infección de transmisión sexual. Una baja proporción de los estudiantes de carreras sanitarias usa constantemente condón, mayormente en las relaciones anales, lo que podría asociarse con una baja percepción de riesgo del no uso de condón.

Palabras clave: condones, salud sexual, estudiantes, educación en salud, enfermedades de transmisión sexual

\begin{abstract}
Introduction: The use of male condoms assists in the prevention of sexually transmitted infections and pregnancy. However, their use among health students has been scarcely studied in Chile. The objective of this study is to evaluate the use of condoms and to characterize the sexual activity and history of sexually transmitted infections among students of the Faculty of Medicine of Universidad de Valparaíso in Chile. Materials and methods: We conducted a cross-sectional study. The Confidential Questionnaire of Active Sexual Life was administered, and the Fisher's exact test and Kruskal-Wallis test were used for inferential analysis. Results: A total of 143 students (69\% women) were included in the study. The students' average age when they first had sexual intercourse was $17+1,8$ years. During the last 12 months, $6.3 \%$ always used a condom, $69.2 \%$ sometimes used a condom, and $24.5 \%$ never used a condom during penetrative sex $(91.6 \%$ of the sexual encounters were heterosexual); in terms of the type of sexual practice, $41.9 \%$ declared always using condoms in anal intercourse, $25.8 \%$ in vaginal, and $0.8 \%$ in oral intercourse; $3.5 \%$ had a history of having a sexually transmitted infection. No associations were found between the use of condom and the participants' sex, history of sexually transmitted infections, average age of their first sexual intercourse, or university career. Discussion: A small number of participants reported a history of sexually transmitted infections. A low proportion of health students of consistently use condoms, with a higher proportion in anal intercourse, which might be related to a low-risk perception of not using a condom.
\end{abstract}

Key words: condoms, sexual health, students, health education, sexually transmitted diseases 


\section{INTRODUCCIÓN}

En Chile, se estima que el $71 \%$ de los jóvenes ha iniciado su actividad sexual a los 16,6 años ${ }^{1,2}$ en promedio, es decir, en un rango etario en que la población es proclive a adoptar comportamientos sexuales de riesgo que se asocian al contagio de infecciones de transmisión sexual (ITS) o a la generación de un embarazo no deseado ${ }^{3}$. En ambos casos nos referimos a problemáticas de salud pública, pues a nivel mundial más de un millón de personas contraen una ITS diariamente ${ }^{4}$ y 87 millones de mujeres experimentan embarazos no deseados cada año ${ }^{5}$. Ambos fenómenos también han sido estudiados desde la perspectiva de los determinantes sociales en salud, puesto que se asocian intrínsecamente a condiciones familiares, sociales, culturales, educacionales, familiares, entre otras. Todas ellas redundan en una inadecuada información respecto a las ITS, el mayor número de parejas sexuales, la práctica frecuente de sexo casual, la baja percepción de riesgo atribuido, el abuso de alcohol u otras sustancias y las presiones del grupo de pares, entre otras ${ }^{6,7}$. No obstante, un hallazgo transversalmente pesquisado es el uso inconsistente de métodos contraceptivos de barrera como el condón, los cuales serían capaces de evitar ambas situaciones descritas.

El condón masculino, mediante su uso sistemátiCo, es capaz de prevenir la transmisión sexual del virus de la inmunodeficiencia humana $(\mathrm{VIH})$ en un $80 \%$ a $95 \%$, reducir el riesgo de contraer otras ITS y prevenir en un $85 \%$ a $98 \%$ los embarazos no desea$\operatorname{dos}^{8}$. No obstante, la frecuencia del uso del condón en las relaciones sexuales entre jóvenes es baja ${ }^{7}$. Uno de los pocos estudios acerca de la frecuencia de uso de condón en población universitaria en Chile, indicó que el 22\% siempre ha usado condón durante las relaciones sexuales, el $52 \%$ a veces y el $26 \%$ nunca ${ }^{9}$, mientras que la Encuesta Nacional de Salud de Chile 2016-2017, reveló que solo el 12,8\% de los hombres y el 7,1\% de las mujeres siempre usó condón durante los últimos 12 meses $^{10}$.

De acuerdo con la Organización de las Naciones Unidas, Chile experimentó un incremento de 34\% en los nuevos casos de infección por $\mathrm{VIH}^{11}$ durante el periodo 2010-2016, de los cuales 40\% se concentró en el grupo etario de 20 a 29 años ${ }^{12}$. En efecto, de acuerdo con el Informe Epidemiológico de las ITS en Chile emitido en $2017^{13}$, el primer lugar de incidencia lo ocupa la sífilis y luego el VIH. En suma, Chile es el país latinoamericano que presen- tó el mayor aumento de nuevos casos de VIH, cuya principal vía de contagio es la sexual. Si bien el mal uso de condón no es el único factor que explica estas cifras, su uso adecuado es un elemento crucial para la prevención de la enfermedad.

Aunque sería plausible plantear que los estudiantes universitarios de carreras de la salud estén más sensibilizados debido a mayores conocimientos técnicos acerca de la importancia del uso del condón, no están exentos de conductas sexuales de riesgo ${ }^{14}$. Pese a la existencia de encuestas e informes nacionales respecto a la situación actual de contagio de ITS en la población chilena, los estudios recientes que aborden esta materia en población universitaria de carreras del ámbito sanitario son muy escasos. En efecto, muy pocos estudios en Chile han indagado en profundidad el uso de condón en población universitaria. Por lo tanto, esta investigación presenta una descripción del uso de condón, de la actividad sexual y del antecedente de ITS en estudiantes de carreras sanitarias en Chile, analizando las asociaciones entre algunas variables.

\section{MATERIALES Y MÉTODOS}

Se realizó un estudio de corte transversal que incluyó a estudiantes de pregrado de entre 18 y 30 años de alguna carrera de la Facultad de Medicina de la Universidad de Valparaíso, Chile, que hayan tenido al menos una relación sexual penetrativa (i.e. vaginal, anal, oral) en los últimos 12 meses. Se efectuó un muestreo no probabilístico que incorporó a todos los estudiantes que cumplieran con los criterios de elegibilidad y quisieran ser parte del estudio. Tras la aplicación del protocolo de consentimiento informado, se empleó el Cuestionario Confidencial Sobre Vida Sexual Activa ${ }^{15}$, diseñado por el Ministerio de Salud de Colombia en 1997. El instrumento ha sido utilizado en diversos estudios que exploraron aspectos de la salud sexual y reproductiva en población joven, demostrando propiedades psicométricas robustas ${ }^{16}$. Adicionalmente, se recabaron datos como curso, edad y carrera universitaria. Para la descripción de datos se utilizaron medias y desviaciones estándar, mientras que en la inferencia estadística se aplicó la prueba exacta de Fisher para comparar proporciones y la prueba de Kruskal-WaIlis para contrastar medias, dada la distribución de las variables. Los datos se analizaron en el programa estadístico STATA 15 (StataCorp, Texas, Estados Unidos), considerando un nivel de significancia del $5 \%$. 
Este estudio fue aprobado por el Comité de Bioética para la Investigación de la Facultad de Medicina de la Universidad de Valparaíso, en el Acta de Evaluación Bioética № 25/2018 el 28 de junio de 2018.

\section{RESULTADOS}

De un total de 205 estudiantes que accedieron a participar en el estudio, 143 cumplieron con los criterios de elegibilidad. Los participantes excluidos correspondieron a estudiantes sin inicio de su actividad sexual o que no habían tenido relaciones sexuales en los últimos 12 meses. El 69\% (n=99) de los participantes fueron mujeres. El promedio de edad fue de $20,8 \pm 2,2$ años y la edad promedio de la primera relación sexual fue de 17+1,8 años. En Tabla 1 se describen estos hallazgos.

Respecto de la frecuencia del uso de condón en relaciones sexuales penetrativas durante los últimos 12 meses, 6,3\% $(n=9)$ de los estudiantes declaró haber usado condón siempre y $24,5 \%(n=35)$ nunca.
De acuerdo con el tipo de práctica sexual penetrativa, $41,9 \% \quad(n=60)$ informó siempre usar condón en las relaciones sexuales anales, $25,8 \%(n=37)$ en las vaginales y $0,8 \%(n=1)$ en las orales. Un $24,5 \%$ $(n=35)$ indicó haber tenido más de una pareja sexual en los últimos 12 meses; por otra parte, 91,6\% $(n=131)$ refirió haber tenido relaciones sexuales penetrativas solo con personas del sexo opuesto y $5,6 \%(n=8)$ con personas del mismo sexo. Finalmente, $3,5 \%(n=5)$ de los estudiantes encuestados señaló haber presentado alguna ITS en los últimos 12 meses. En Tabla 2 se presentan estos resultados.

No se encontraron asociaciones estadísticamente significativas al analizar la relaciones entre la uso de condón en relaciones sexuales vaginales, anales y orales; y sexo del estudiante, antecedente de ITS, sexo de la pareja, proporción de estudiantes con una o más parejas sexuales, promedio de edad de la primera relación sexual o carrera universitaria del participante.

Tabla 1. Características generales de los participantes.

\begin{tabular}{l|l}
\hline Características & $\mathbf{n}=\mathbf{1 4 3}$ \\
\hline Edad & $20,8(+2,2$ años $)$ \\
\hline Cantidad de mujeres & $99(69,2 \%)$ \\
\hline Edad de la primera relación sexual & $17(+1,8$ años) \\
\hline Cantidad de estudiantes de medicina & $32(22,4 \%)$ \\
\hline Cantidad de estudiantes de fonoaudiología & $25(17,5 \%)$ \\
\hline Cantidad de estudiantes de kinesiología & $23(16,1 \%)$ \\
\hline Cantidad de estudiantes de obstetricia y puericultura & $23(16,1 \%)$ \\
\hline Cantidad de estudiantes de tecnología médica & $15(10,5 \%)$ \\
\hline Cantidad de estudiantes de enfermería & $14(9,8 \%)$ \\
\hline Cantidad de estudiantes de educación parvularia & $11(7,6 \%)$ \\
\hline Cantidad de estudiantes de educación parvularia & $11(7,6 \%)$ \\
\hline
\end{tabular}

*Resultados en media (desviación estándar) o número absoluto (proporción). 
Tabla 2. Caracterización de la vida sexual de los participantes según uso de condón, sexo de la pareja y antecedente de ITS.

\begin{tabular}{|c|c|c|c|}
\hline Variable & $n=143$ & & \\
\hline \multicolumn{4}{|c|}{ Uso de condón en todas las relaciones sexuales } \\
\hline & Siempre & 9 & $(6,3 \%)$ \\
\hline & A veces & 99 & $(69,2 \%)$ \\
\hline & Nunca & 35 & $(24,5 \%)$ \\
\hline \multicolumn{4}{|c|}{ Antecedente de ITS } \\
\hline & Presente & 5 & $(3,5 \%)$ \\
\hline & Ausente & 127 & $(88,8 \%)$ \\
\hline & Desconocido & 11 & $(7,7 \%)$ \\
\hline \multicolumn{4}{|c|}{ Sexo de la pareja } \\
\hline & Mismo sexo & 8 & $(5,6 \%)$ \\
\hline & Sexo opuesto & 131 & $(91,6 \%)$ \\
\hline & Ambos sexos & 4 & $(2,8 \%)$ \\
\hline \multicolumn{4}{|c|}{ Número de parejas } \\
\hline & Única & 108 & $(75,5 \%)$ \\
\hline & Más de una & 35 & $(24,5 \%)$ \\
\hline \multicolumn{4}{|c|}{ Uso de condón en relaciones sexuales vaginales } \\
\hline & Siempre & 37 & $(25,8 \%)$ \\
\hline & A veces & 74 & $(51,5 \%)$ \\
\hline & Nunca & 32 & $(22,5 \%)$ \\
\hline \multicolumn{4}{|c|}{ Uso de condón en relaciones sexuales anales } \\
\hline & Siempre & 60 & $(41,9 \%)$ \\
\hline & A veces & 32 & $(22,6 \%)$ \\
\hline & Nunca & 51 & $(35,5 \%)$ \\
\hline \multicolumn{4}{|c|}{ Uso de condón en relaciones sexuales orales } \\
\hline & Siempre & 1 & $(0,8 \%)$ \\
\hline & A veces & 24 & $(16,4 \%)$ \\
\hline & Nunca & 118 & $(82,8 \%)$ \\
\hline
\end{tabular}

*Resultados en número absoluto (proporción). 


\section{DISCUSIÓN}

Los resultados de este estudio muestran que, en estudiantes de la Facultad de Medicina de la Universidad de Valparaíso, la primera relación sexual ocurrió en promedio a los 17 años. Durante los últimos 12 meses, $6,3 \%$ de los participantes siempre usó condón en las relaciones sexuales penetrativas y $91,6 \%$ de ellas ocurrieron con personas del sexo opuesto. Solo 3,5\% comentó haber presentado alguna ITS en los últimos 12 meses.

En la muestra estudiada, la proporción de personas que usaron constantemente condón es inferior a lo reportado en datos nacionales ministeriales ${ }^{17}$ y en otras muestras de estudiantes universitarios latinoamericanos ${ }^{14,18}$, ya que en este último caso las cifras son cercanas al $30 \%$. Por lo general, el uso constante de condón en estudiantes universitarios es heterogéneo ${ }^{19-21}$, con un predominio claro de prácticas sexuales de riesgo ${ }^{21}$. Sin embargo, las fuentes no suelen especificar la frecuencia del uso de condón según el tipo de relación sexual, por lo que sus resultados podrían ser poco específicos. Aun así, los hallazgos observados en nuestro análisis son preocupantes si se considera que se investigó a una población que debería tener un nivel mayor de conocimiento respecto a los beneficios del uso del preservativo, ya que todos fueron estudiantes de alguna carrera del área de la salud. En ellos, se esperaría que algunos factores cognitivos y socioculturales se vincularan positivamente con las creencias y el uso de preservativos, pues aquí se consolidaría la dinámica y la práctica de la sexualidad ${ }^{22}$. Por ende, es necesario fomentar de manera concordante una cultura de responsabilidad y autocuidado de la salud sexual. Si bien se ha reconocido que la educación sexual durante el periodo escolar es sumamente relevante, los estudios dedicados a abordar las técnicas educativas y los programas de educación sexual implementados actualmente en Chile son insuficientes, lo que ha sido manifestado tanto por los estudiantes y los investigadores en el ámbito. Del mismo modo, los contenidos de los programas y las intervenciones en educación sexual a nivel secundario y superior se han concentrado sobre todo en el embarazo, olvidando aspectos gravitantes del desarrollo psicosexual como el conocimiento y la relación con la propia corporalidad así como con el placer, por lo que se ha enfatizado en mover el foco desde la educación en riesgo sexual hacia la educación en salud sexual ${ }^{23-25}$, ya que permitiría una comprensión integradora de la sexualidad y sería un enfoque práctico en cuanto a la reducción de desenlaces como las ITS y el embarazo no deseado ${ }^{25,26}$.

Algunas barreras para el uso de condón informadas por los estudios publicados son tener relaciones monógamas, falta de experiencia o fracaso al utilizarlo, vergüenza al comprarlos, consumir alcohol u otras drogas y la creencia acerca de la pérdida de placer o espontaneidad; no obstante, la mayor barrera sería la baja percepción de riesgo respecto de las ITS 18,19 . Otros factores individuales asociados al bajo uso de preservativo son la sensación de "invulnerabilidad" característica de esta etapa del ciclo vital $^{21,27}$. Contrariamente, algunas investigaciones demuestran que la percepción de autoeficacia y la asertividad sexual incrementan el uso de condón en población joven ${ }^{28}$. Por otra parte, una interpretación sociológica argumenta que en Latinoamérica las bajas tasas de uso de condón están ligadas al aumento en las libertades sociales vividas por estas naciones durante las últimas décadas ${ }^{29}$.

La proporción más baja del uso de condón se dio en las relaciones sexuales penetrativas orales, donde se observó una frecuencia muy baja $(0,8 \%)$, lo cual es congruente con lo encontrado en un estudio realizado en México similar al presente ${ }^{14}$. Los resultados podrían explicarse, nuevamente, por la menor percepción de riesgo de trasmisión de ITS mediante esta práctica en particular ${ }^{30}$.

No se identificó una asociación significativa entre la frecuencia del uso de condón en los últimos 12 meses y la edad de la primera relación sexual, haIlazgo que coincide con un estudio que, mediante un modelo de regresión logística, determinó que la edad de la primera relación sexual no era una variable que predijera significativamente el tener relaciones sexuales de riesgo ${ }^{14}$. Asimismo, no fue posible pesquisar una asociación entre la frecuencia de uso de condón y el antecedente de ITS, lo que podría relacionarse con la baja proporción de ITS reportadas. Sin embargo, y pese a que la evidencia ha demostrado consistentemente que el uso de condón reduce la probabilidad de una ITS (e.g. reducción de la infección por VIH hasta en un $80 \%{ }^{31}$ ), aún existe una baja percepción de riesgo de las relaciones sexuales en cuanto a su contagio. Pese a esto, algunos reportes han corroborado que el uso del condón en población joven se vería justificado sobre todo para evitar embarazos ${ }^{32}$, relegando a un segundo plano la importancia de las ITS.

La relación entre sexo y uso de condón es un ha- 
llazgo discordante en los artículos publicados. Un estudio realizado en población adolescente y adulta joven describió que la frecuencia del uso del condón en las mujeres era inferior a la de los hombres ${ }^{33}$, mientras que otra investigación conducida en población universitaria, verificó que el uso del condón en la primera relación sexual, en la pareja formal y en la ocasional, era superior en mujeres que en hombres $^{7}$. En este sentido, algunos autores han puntualizado que el tener una pareja estable se asocia a un menor uso de condón ${ }^{7,33}$, lo que no se corroboró en nuestra investigación. En cuanto al sexo de la pareja, la literatura ha reportado que el uso de condón difiere en hombres que tienen sexo con hombres y hombres que tienen sexo con mujeres ${ }^{34}$, pero en nuestro estudio no se determinó una asociación entre ambas variables. Esto podría explicarse por un insuficiente tamaño muestral. Tampoco se halló una asociación significativa entre la frecuencia del uso de condón y la carrera estudiada.

Dentro de las debilidades de este estudio, el bajo tamaño de la muestra podría haber provocado que algunos grupos minoritarios se vieran subrepresentados, tales como el grupo de hombres que tiene sexo con hombres, lo que dificulta el estudio de la inferencia estadística. Sería importante ampliar la cantidad de participantes estudiados, incorporando también a estudiantes de carreras distintas a las de la salud. De este modo, podría conocerse una realidad más amplia que integre perspectivas provenientes de estudiantes universitarios distintos. Otros diseños observacionales como los estudios de casos y controles y los estudios de cohorte permitirían precisar los factores específicos asociados a desenlaces como las ITS o los embarazos no deseados, mientras que el desarrollo de estudios experimentales posibilitaría seleccionar la mejor intervención educativa, por ejemplo, para evitarlos.

Esta investigación contribuye a la muy escasa suma de estudios realizados en Chile sobre el uso de condón en estudiantes universitarios, más aún en estudiantes de carreras sanitarias. Si bien no se establecieron asociaciones estadísticas significativas entre las variables estudiadas, se caracterizó de modo específico el uso de condón en función de cada práctica sexual, obteniéndose resultados que concuerdan con el patrón general referido por las publicaciones disponibles, lo que refuerza la importancia de la temática. A futuro, sería interesante indagar en las motivaciones a la base del uso del condón, lo que posibilitaría una comprensión más acabada del fenómeno y la implementación de estrategias que fomenten el uso de preservativos.

\section{Fuente de apoyo económico}

Los autores declaran que esta investigación no tuvo fuentes de apoyo económico.

\section{Conflicto de interés}

Los autores declaran no tener algún conflicto de intereses.

\section{REFERENCIAS BIBLIOGRÁFICAS}

1. Instituto Nacional de la Juventud (Chile). Octava Encuesta Nacional de Juventud. Santiago, Chile: INJUV; 2015.

2. Severino-Vergara A, Velásquez-Muñoz A, Rivera-Marín J, González-Araya E. Anticoncepción en la adolescencia: motivaciones, elecciones y educación sanitaria en un centro de medicina reproductiva de Chile. Matronas Prof. 2017;18(4):144-51.

3. Espada J, Quiles M, Méndez J. Conductas sexuales de riesgo y prevención del SIDA en la adolescencia. Papeles del psicólogo. 2003;24(85):29-36.

4. World Health Organization. Report on global sexuaIly transmitted infection surveillance, 2018 [Internet]. Geneva: WHO; 2018 [consultado en sept 2019]. Disponile en: https://www.who.int/reproductivehealth/publications/stis-surveillance-2018/en/

5. Bellizzi S, Sobel HL, Obara H, Temmerman M. Underuse of modern methods of contraception: underlying causes and consequent undesired pregnancies in 35 low- and middle-income countries. Hum Reprod. 2015;30(4):973-86.

6. Isaac Uribe J, Covarrubias Cuellar KY, Andrade Palos P. La cultura sexual de los adolescentes colimenses aspectos característicos de la cultura local. Estud Cult Contemp. 2008;14(28):61-95.

7. Uribe J, Amador G, Zacarías X, Villarreal L. Percepciones sobre el uso del condón y la sexualidad entre jóvenes. Rev Latinoam Cienc Soc Niñez Juv. 2012;10(1):481-94.

8. Organización Mundial de la Salud. Planificación familiar: un manual mundial para proveedores. Ginebra: OPS; 2011.

9. Espinoza E, Quinteros N. Comportamiento sexual en las y los estudiantes de pregrado del Campus Isla Teja de la Universidad Austral de Chile, 2008. Valdivia: Universidad Austral de Chile; 2008. 
10. Chile. Ministerio de Salud. Encuesta Nacional de Salud 2016-2017. Santiago: MINSAL; 2017.

11. Organización de las Naciones Unidas. Ending AIDS. Progress towards the 90-90-90 targets [Internet]. Ginebra: ONU; 2017 [consultado en agosto 2019]. Disponibe en: https://www.unaids.org/sites/default/files/ media_asset/Global_AIDS_update_2017_en.pdf

12. Chile. Ministerio de Salud. Resultados confirmación de infección por VIH en Chile, 2010-2015 [Internet]. Santiago: MINSAL; 2016 [consultado en sept 2019]. Disponible en: http://www.ispch.cl/sites/default/files/ BoletinVIH-15112017A.pdf

13. Cáceres-Burton Karen. Report: Epidemiological situation of sexually transmitted infections in Chile, 2017. Rev. chil. infectol. [Internet]. 2019 Apr [consultado en oct 2019];36(2):221-233. Disponible en: https:// scielo.conicyt.cl/scielo.php?script=sci_arttext\&pi$\mathrm{d}=$ S0716-10182019000200221\&lng=en. http://dx. doi.org/10.4067/S0716-10182019000200221

14. Staines H, Fraga M, Menchaca R, Salazar J, Vargas A, Bucardo J, et al. Actitudes sexuales y uso del condón en estudiantes universitarios de Ciudad de Juárez, México. Tecnociencia Chihuahua. 2009;3(2):84-96.

15. Colombia. Ministerio de Salud. Cuestionario confidencial sobre vida sexual activa. Santa Fe de Bofotá; 1997.

16. Contreras F, Carboles JA, Juárez-Acosta F. Propiedades psicométricas del cuestionario confidencial de vida sexual activa para evaluar conductas de riesgo en VIH-SIDA. Psicol Conduct. 2007;15(1):5-27.

17. Chile. Ministerio de Salud. Encuesta de Calidad de Vida y Salud (ENCAVI) 2015-2016. Santiago; 2017.

18. Fraga M, Dávila W, Vargas-Ojeda A, Bucardo J, Patterson-Thomas L, Staines H. Uso del condón en estudiantes universitarios de Tijuana. Expresiones Médicas. 2008;4(3):95-104.

19. Orcasita L, López M, Reina C. Conocimientos sobre riesgos frente a infecciones de transmisión sexual (ITS) en estudiantes universitarios de la ciudad de Cali. Inf Psicológicos. 2014;14(1):143-58.

20. Álvarez L, Tamayo L, Moreno M. Conocimientos y prácticas en salud sexual de los estudiantes de la Universidad de Antioquia, Medellín, Colombia, 2014. Hechos Microbiol. 2014;5(1):25-35.

21. Fernández-Silva C, Sánchez-Martínez D. Actitudes y prácticas sexuales relacionadas con VIH/SIDA. Rev Univ Ind Santander Salud. 2018;50(4):352-7.

22. Moral de la Rubia J, Ortega M. Diferencias de géne- ro en significados, actitudes y conductas asociados a la sexualidad en estudiantes universitarios. Estud Cult Contemp. 2008;14(28):97-119.

23. Apter D, Molina-Cartes R. Sexuality Education: Finnish and Chilean Experiences. Endocr Dev. 2012;22:332-56.

24. Obach A, Sadler M, Jofré N. Salud sexual y reproductiva de adolescentes en Chile: el rol de la educación sexual. Rev Salud Publica. 2017;19(6):848-54.

25. Kantor LM, Lindberg L. Pleasure and sex education: The need for broadening both content and measurement. Am J Public Health. 2020;110(2):145-8.

26. Koepsel ER. The power in pleasure: Practical implementation of pleasure in sex education classrooms. Am J Sex Educ. 2016;11(3):205-65.

27. Rojas-Murcia C, Pastor Y, Esteban-Hernández J. Ilusión de invulnerabilidad, estereotipos y percepción de control del SIDA en universitarios. Rev Iberoam Psicol Salud. 2015;6(1):28-38.

28. Uribe-Alvarado J, Bahamón M, Reyes-Ruiz L, Trejos-Herrera A, Alarcón-Vásquez Y. Percepción de autoeficacia, asertividad sexual y uso del condón en jóvenes colombianos. Act Colom Psicol. 2017;20(1):203-11.

29. Ramírez-Correa P, Ramírez-Santana M. Predicting condom use among undergraduate students based on the theory of planned behaviour, Coquimbo, Chile, 2016. Int J Env Res Public Heal. 2018;15(8):1689.

30. Godoy-Flores F, Lee-Muñoz X. Conocimientos, conductas y motivos en los métodos preventivos para prácticas sexuales ororeceptivas en estudiantes de odontología. Rev Clin Periodoncia Implant Rehabil Oral. 2019;12(1):23-6.

31. Weller SC, Davis-Beaty K. Condom effectiveness in reducing heterosexual HIV transmission. Cochrane Database Syst Rev. 2002;(1):CD003255.

32. Uribe J, Carrillo S, Riaño M, Bonilla-Cruz N. Expresiones de la sexualidad y del uso del condón en las relaciones sexuales en jóvenes universitarios de Colombia. Aportes a la clínica psicológica. AVFT. 2018;37(5):456-62.

33. Soto V. Factores asociados al no uso del condón: estudio en adolescentes y adultos jóvenes de Chiclayo. An Fac Med. 2006;67(2):152-9.

34. Wildman L, Golin C, Grodensky C, Suchindran C. Do safer sex self-efficacy, attitudes toward condoms, and HIV transmission risk beliefs differ among men who have sex with men, heterosexual men, and women living with HIV? AIDS Behav. 2013;17(5):1873-82. 